\title{
Reproductive performance in sows in relation to Japanese Encephalitis Virus seropositivity in an endemic area
}

\author{
Johanna Lindahl • Sofia Boqvist • Karl Ståhl • \\ Ho Thi Viet Thu $\cdot$ Ulf Magnusson
}

Accepted: 27 October 2011 /Published online: 12 November 2011

(C) The Author(s) 2011. This article is published with open access at Springerlink.com

\begin{abstract}
Japanese Encephalitis Virus (JEV) is considered an important reproductive pathogen in pigs. Most studies of the reproductive impact of JEV have been conducted in areas where the disease occurs in seasonal epidemics. In this study, the associations between seropositivity for JEV, measured with an IgG ELISA, and the number of piglets born alive and stillborn were investigated in a tropical area endemic for JEV in Vietnam. Sixty percent of sows from four farms in the Mekong delta of Vietnam were seropositive to JEV and the Odds Ratio for a sow being infected was highest (6.4) in sows
\end{abstract}

J. Lindahl $\cdot$ U. Magnusson $(\bowtie)$

Department of Clinical Sciences, Division of Reproduction, Swedish University of Agricultural Sciences, P.O. Box 7054, SE-750 07 Uppsala, Sweden e-mail: ulf.magnusson@slu.se

J. Lindahl

e-mail: Johanna.lindahl@slu.se

S. Boqvist $\cdot$ K. Ståhl

Department of Biomedicine and Veterinary Public Health, Swedish University of Agricultural Sciences, P.O. Box 7009, SE-750 07 Uppsala, Sweden

\section{S. Boqvist \\ e-mail: Sofia.Boqvist@slu.se \\ K. Ståhl \\ e-mail:karl.stahl@slu.se \\ K. Ståhl \\ National Veterinary Institute, \\ SE-751 89 Uppsala, Sweden}

\section{H. T. V. Thu}

Department of Veterinary Medicine, Campus II,

Can Tho University,

Can Tho, Vietnam

e-mail: htvthu@ctu.edu.vn above 3.5 years (95\% confidence interval 2.2-18.3). There was an association between increasing Optical Density (OD) values from the ELISA and the number of stillborn piglets in sows less than 1.5 years, but no effect of seropositivity could be shown when all sows were studied. OD values had an effect $(p=0.04)$ on the number of piglets born alive in the statistical analysis only when interacting with the effect of the breeds. An increase in mean OD value of the herd was correlated $(p<0.0001)$ with an increase in the number of piglets born alive. In this study, there was evidence of a negative association between seropositivity for JEV and the reproductive performance only in sows less than 1.5 years in endemic areas. This could be explained by a year-round infection with the virus, which would lead to immunity in many gilts before their first pregnancy. This, in turn, may imply that JEV infection in pigs is of minor importance for the reproductive performance in endemic areas.

Keywords Zoonosis · Vector borne · Flavivirus · Pig · Reproduction $\cdot$ Emerging disease

\section{Introduction}

Japanese Encephalitis Virus (JEV) is a well-known zoonotic agent causing viral encephalitis in humans and is also known to affect the reproductive performance of pigs. Similar to other viral pathogens infecting fetuses in utero, JEV can cause stillbirth, mummification, embryonic death and infertility in pigs (Salmon 1984; Gresham 2003). The first association between JEV and reproductive failure in pigs was observed in 1947-1948 in Japan when there were large outbreaks of encephalitis in humans and horses, concomitantly with outbreaks of stillbirths in pigs, and JEV was subsequently 
isolated from stillborn piglets (Hosoya et al. 1950; Burns 1950). The virus is considered an economically important reproductive pathogen of pigs due to production losses (Platt and Joo 2006). However, most studies on the reproductive impact of JEV in pigs were performed several decades ago and there is little new data on this topic.

Different mosquito species in the family Culicidae serve as vectors for the virus (Rosen 1986) and the transmission is thus dependent on the vector species existing in an area and the season in which these are active. The virus has emerged to new areas over the past half-century and is now reported from large parts of South and East Asia and northern Australia (Hanna et al. 1996; Endy and Nisalak 2002). Being a vector-borne pathogen, there is a risk that the emergence of JEV will be enhanced by the climate changes (Gubler 1996; Khasnis and Nettleman 2005).

Most studies on the reproductive impact of JEV in pigs have been made in areas where vectors are present seasonally and the disease pattern is epidemic. Less is known about the importance of the infection in areas where vectors are active all year around and the disease is endemic. Endemic transmission of JEV occurs mostly in countries in tropical latitudes (Endy and Nisalak 2002). Given the emergence of the disease and climate changes, an endemic situation might be more common in the future and knowledge about the impact of JEV on the pig industry under these new circumstances is needed.

Here, we investigate the association between seropositivity for JEV in sows in the Mekong delta in Vietnam and their reproductive performances. This area has an intense production of swine (Hai and Nguyen 1997) and JEV is reported to be endemic (Do et al. 1994; Nguyen and Nguyen 1995; Bartley et al. 2002).

\section{Materials and methods}

Study area

The sampling was conducted in the Mekong delta in the south of Vietnam in 1999 (Boqvist et al. 2002). This region has two seasons: the wet season between May and
November, and the dry season between December and April (Minh et al. 1997). Traditionally, the Mekong delta is one of the most highly pig-producing regions in Vietnam and production has increased over the last part of the 20th century (Vo-Tong et al. 1995; Hai and Nguyen 1997). The majority of the pig farms are small-scale family farms with 1-2 sows, with a growing number of larger commercial farms and some large state-owned farms, which may have many hundred sows.

Study population and study design

The study population and the samples used in this study have previously been described by Boqvist et al. (2002), and are briefly reviewed here. Four state-owned pig farms, labeled A-D, located in the Mekong delta of Vietnam were selected. All farms reported routine vaccinations against Foot and Mouth Disease and Classical Swine Fever, farms $\mathrm{B}$ and $\mathrm{D}$ also reported vaccination against Aujeszky's disease, and all, except for farm $\mathrm{C}$, reported vaccination against Porcine Parvovirus. All farms wean the piglets at 4 weeks. Farms were visited at the end of the dry period (March), at the middle of the rainy period (August) and at the beginning of the dry period (December).

Within the farms, sows in the second month of gestation or during lactation were selected. A maximum of 20 sows were included from each of the two reproductive stages at each of the three visits and no sow was sampled on more than one occasion. The distribution of animals used in this study, per farm and sampling occasion is shown in Table 1.

\section{Sampling and data collection}

Pigs were bled from the jugular vein, the blood samples were allowed to clot centrifuged at 2,000 rpm and the sera were kept cool during local and international transport and frozen during storage (Boqvist et al. 2002). The sera were inactivated at $56^{\circ} \mathrm{C}$ for $30 \mathrm{~min}$ and then stored at $-20^{\circ} \mathrm{C}$ until analyzed.

Animal and reproductive data was collected through written questionnaires containing both open and closed questions. The questionnaires were given to the farm
Table 1 The number of sows at four state farms in the Mekong delta in Southern Vietnam sampled for serological analysis of Japanese Encephalitis Virus

\begin{tabular}{llllll}
\hline & \multicolumn{2}{l}{ Farm } & & & Total \\
\cline { 2 - 5 } & A & B & C & D & \\
\hline Number of sows sampled in March & 40 & 6 & 32 & 35 & 113 \\
August & 34 & 5 & 32 & 33 & 104 \\
December & 36 & 10 & 22 & 30 & 98 \\
Number of sows sampled in total & 110 & 21 & 86 & 98 & 315 \\
Total number of sows on farm & 434 & 53 & 143 & 200 & 830 \\
\hline
\end{tabular}


managers on one visit to be collected at a later occasion. For pregnant sows, data was collected for the litter born after the sampling and for lactating sows for the present litter. The date of sampling was recorded and the data collected included age, parity number, age at first litter, breed, week of lactation or pregnancy, the number of piglets born in total, the number of piglets born alive, the weaning to service interval and the number of aborted or mummified fetuses. The number of stillborn piglets was calculated as the difference between the number of total born and the number of live born piglets. Serological status against Leptospira from earlier studies (Boqvist et al. 2002) was also included. The distribution of age, breed and Leptospira status per farm is shown in Table 2.

\section{Serological analyses}

Samples were analyzed for antibodies against JEV using a commercial indirect IgG enzyme-linked immunosorbent assay (ELISA) (Shenzhen Lvshiyuan Biotechnology Co. Ltd., Shenzhen, China) according to manufacturer's instructions. Based on data provided by the manufacturer, the assay had a relative specificity of 0.79 and a relative sensitivity of 0.92 compared to Hemagglutination Inhibition test (HI). Additional data provided by the manufacturer showed good reproducibility and no cross-reaction with antibodies to other significant pathogens such as Porcine

Table 2 The distribution by age, breed and serology of sows sampled for serological analysis of Japanese Encephalitis Virus at four state farms in the Mekong delta in Southern Vietnam

\begin{tabular}{lllllll}
\hline Variable & \multicolumn{1}{l}{ Farm } & & & & Total & (\% of known) \\
\cline { 2 - 4 } & A & B & C & D & & \\
\hline Age & & & & & & \\
$<1.5$ year & 30 & 0 & 4 & 23 & 57 & $(21)$ \\
$1.5-3.5$ years & 55 & 10 & 57 & 58 & 180 & $(65)$ \\
$>3.5$ years & 23 & 0 & 8 & 10 & 41 & $(15)$ \\
Unknown & 2 & 11 & 17 & 7 & 37 & \\
Breed & & & & & & \\
Duroc & 2 & 0 & 10 & 3 & 15 & $(5)$ \\
Landrace & 14 & 1 & 13 & 0 & 28 & $(10)$ \\
Yorkshire & 43 & 16 & 30 & 75 & 164 & $(59)$ \\
Hybrids & 49 & 4 & 16 & 3 & 72 & $(26)$ \\
Unknown & 2 & 0 & 17 & 17 & 36 & \\
Leptospira & & & & & & \\
Negative & 46 & 15 & 52 & 47 & 160 & $(52)$ \\
Positive & 64 & 6 & 31 & 49 & 150 & $(48)$ \\
Unknown & 0 & 0 & 3 & 2 & 5 & \\
\hline
\end{tabular}

${ }^{\text {a }}$ A sow is considered positive for Leptospira when tested seropositive for the serovars bratislava, grippotyphosa, icterohemorrhagiae, pomona or tarassovi (Boqvist et al. 2002)
Parvovirus and Porcine reproductive and respiratory syndrome virus. The same data was published by Xinglin et al. (2005) in the development of an IgG ELISA.

To further control the specificity of the test, 50 serum samples from Swedish pigs were tested and 18 of these were inactivated by $56^{\circ} \mathrm{C}$ for 30 and $60 \mathrm{~min}$ to study the effect of heat inactivation on the results. All these samples were negative indicating a $100 \%$ specificity of the test in samples from pigs not expected to have been infected with Flavivirus. No increase in OD values was seen after inactivation of the serum.

According to the manufacturer's instructions, Optical Density (OD) values below 0.38 were considered negative, above 0.42 positive and values between and equal to 0.38 and 0.42 were considered inconclusive. Since it has been shown that OD values and $\operatorname{IgG}$ antibody titers are well correlated in other flaviviral infections (Tardei et al. 2000) and antibody titers have been observed to be correlated with clinical outcome in some viral diseases (Ho et al. 2005; Hung et al. 2010), the serological results were handled both as continuous OD values and as a dichotomous outcome, either positive or negative. Inconclusive results were considered missing values in all analyses where the ELISA results were used as a dichotomous variable.

\section{Statistical analyses}

Statistical analyses were performed using SAS for Windows 9.2 (SAS Institute Inc., Cary, NC, USA). Univariable analyses on the differences between farms, regarding the seroprevalence and the reproductive parameters, were performed using the univariate, frequency and the mixed procedures.

Correlations between the variables age, parity number, breed, month of sampling, seropositivity for Leptospira, and farm were investigated using Spearman correlation test in the correlation procedure. The variables age and parity were correlated $(\mathrm{Sp}=0.92)$ and parity was excluded as age was considered to be more relevant for the possibility to be seropositive.

In the analyses of serological data, associations between the ELISA results as the dependent variable and the variables age, farm, breed and month of sampling were investigated using the Glimmix procedure for logistic regression with farm included as a random effect. In this analysis, the ELISA results were dichotomous and either negative or positive. All variables were included and eliminated using backward elimination until all remaining variables showed $p \leq 0.05$, since this method allows for good assessment of potential confounders (Dohoo et al. 2009). Interactions between these variables were then tested and eliminated the same way.

In the analyses of reproductive performance, two dependent variables, the numbers of piglets born alive and 
stillborn, were investigated. Due to low response rate $(<70 \%)$ for the number of aborted and mummified fetuses and weaning to service interval, these variables were not analyzed further. The number of piglets born alive showed a normality distribution as assessed by the univariate procedure and the number of stillborn piglets showed a negative binomial distribution confirmed by the fit statistics (Pearson $\chi^{2} /$ degree of freedom) in the Glimmix procedure. Analyses were performed first with all sows and then including only the youngest category of sows ( $<1.5$ years).

To investigate associations between the number of piglets born alive and the serological results, age, month of sampling, breed and seropositivity for Leptospira, general linear regression in the mixed procedure was used. The Glimmix procedure was used to investigate associations between the number of stillborn piglets and the variables mentioned above. All these analyses were performed using both the dichotomous outcome and the continuous OD values from the ELISA.

In all models for reproductive performance, the effect of farm was included as a random effect in the models except in the one for stillborn piglets in sows less than 1.5 years and dichotomous serological results when farm was included as a fixed effect. The serological results were forced to stay in the models. The remaining four categorical variables were eliminated using backward elimination until the remaining variables showed $p \leq 0.05$. The variables age, breed and month of sampling were tested as potential confounders in both models. Month of sampling could be a potential confounder since it was correlated to the time of farrowing, which earlier has been shown to affect the reproductive performance of sows (Tantasuparuk et al. 2000). Age and month of sampling were included as confounders in the model for the number of piglets born alive as they changed the estimate with more than $25 \%$. Interactions between all variables thus kept in the models were tested and kept in the final model if they showed $p \leq 0.05$. When a significant interaction with OD values was found, a nested model was performed, which estimated the effects of OD values within each category.

The analysis of the number of piglets born alive using the continuous OD values was also repeated with the OD value mean of the farm included and the individual values were added as a deviance from the mean in order to test for contextual effects.

\section{Results}

Serological results

Sixty percent (190/315) of the sampled sows were tested seropositive for JEV, $37 \%$ (116/315) were seronegative and $3 \%(9 / 315)$ showed inconclusive results. The median OD value of all 315 sows was 0.67 and the mean was 0.82 .

The percentage of seropositive animals and the mean OD values differed significantly $(p<0.0001)$ between farms in the univariate analysis. In the multivariable analysis, the only factor with a significant impact on the odds for being seropositive was age $(p=0.002)$. Compared to sows under the age of 1.5 years of age, sows between 1.5 and 3.5 years had an Odds Ratio (OR) of 2.4 (95\% confidence interval 1.2-4.8) and sows older than 3.5 years had an OR of 6.4 (95\% confidence interval 2.2-18.3) for being seropositive.

\section{Reproductive performance in all sows}

The average number of piglets born in total was 10.1 and number of piglets born alive was 9.2 per sow. There were significant differences between the farms regarding the number of piglets born in total $(p<0.0001)$, the number of piglets born alive $(p<0.0001)$ and the number of stillborn piglets $(p=0.0009)$. The range and average per farm is shown in Table 3.

Breed was the only predictor with an effect $(p=0.006)$ in itself and in interaction with the effect of age $(p=0.01)$ when comparing the number of piglets born alive between seropositive and seronegative sows. There was no association between the number of stillborn piglets and serological results as positive or negative.

The effect of OD values on the number of piglets born alive was only significant when interacting with the effect of the breeds $(p=0.04)$. Because of this, the effects of the OD values were estimated within the breeds (Tables 4 and 5). Duroc and hybrids had an increase of 0.8 piglets born alive for every one-unit increase in OD value, whereas
Table 3 Average number of piglets born in total, alive and stillborn per sow at four state farms in the Mekong delta in Southern Vietnam

\begin{tabular}{|c|c|c|c|c|c|c|c|c|c|c|}
\hline \multirow[t]{2}{*}{ Piglets born } & \multicolumn{8}{|c|}{ Farm } & \multirow[t]{2}{*}{ Total } & \multirow[t]{2}{*}{ Range } \\
\hline & A & Range & B & Range & $\mathrm{C}$ & Range & $\mathrm{D}$ & Range & & \\
\hline Total & 11.1 & (4-19) & 11.1 & $(10-13)$ & 8.6 & $(3-14)$ & 10.1 & $(5-15)$ & 10.1 & $(3-19)$ \\
\hline Alive & 10.2 & $(1-15)$ & 10.7 & $(10-12)$ & 7.2 & $(2-11)$ & 9.6 & $(5-14)$ & 9.2 & $(1-15)$ \\
\hline Stillborn & 0.9 & $(0-9)$ & 0.4 & $(0-2)$ & 1.4 & $(0-9)$ & 0.5 & $(0-2)$ & 0.9 & $(0-9)$ \\
\hline No data & 3 & & 1 & & 1 & & 12 & & 17 & \\
\hline
\end{tabular}


Table 4 Association between the serology for Japanese Encephalitis Virus as continuous OD values and the number of piglets born alive

\begin{tabular}{lll}
\hline Effect & $F$ value & $P$ \\
\hline Within breed effect of OD values & 2.16 & 0.0739 \\
Breed & 1.97 & 0.1188 \\
Age & 1.07 & 0.3430 \\
Season & 0.59 & 0.5530 \\
Interaction between age and breed & 3.42 & 0.0030 \\
\hline
\end{tabular}

Landrace and Yorkshire showed a decrease of 1 and 0.5 piglets born alive, respectively. Although there were significant differences between breeds, none of the estimates were in themselves significantly different from zero.

When the mean OD value of the herds was included in the analysis, there was no effect of the individual serological result on the number of piglets born alive. The mean OD value of the herds had an effect $(p<0.0001)$ with an estimated increase of 5.2 piglets born alive (standard error 1.1) for every one-unit increase in mean OD value for the herd. There was still an effect of breed $(p=0.009)$ and breed and age interaction $(p=0.01)$.

Data on abortions and mummifications were available for 211 sows. There were only four observations of abortions in the data and all of these sows were seropositive. The maximum number of mummified fetuses reported from a sow was five, and in total there were only nine sows with more than two mummified fetuses born. The weaning to service interval, ranged between 2 and 40 days with a mean interval of 6.9 days. There was data on 215 sows.

Reproductive performance in sows less than 1.5 years

In the sows less than 1.5 years, the serological status $(p=0.049)$ and the breed $(p=0.002)$ were associated with the number of stillborn piglets when OD values were continuous. There was an estimated increase of 0.88 stillborn piglets for every oneunit increase in OD value. This association could not be seen when animals were considered serologically positive or negative only.

Table 5 Association between the serology for Japanese Encephalitis Virus results and the number of piglets born alive within each breed

\begin{tabular}{lcc}
\hline Breed & Coefficient $^{\mathrm{a}}$ & Standard error \\
\hline Duroc & 0.76 & 1.40 \\
Landrace & -1.06 & 0.68 \\
Yorkshire & -0.48 & 0.32 \\
Hybrids & 0.83 & 0.46
\end{tabular}

${ }^{\mathrm{a}}$ The coefficient shows how a one-unit increase in OD value affects the number of piglets born alive in each breed
There were significant associations between the number of piglets born alive and the interactions between breed and serological results, both when serology was handled as continuous ( $p=0.043)$ and dichotomous $(p=0.032)$ variables. The results when the continuous OD values were used in the model are shown in Tables 6 and 7.

The average number of piglets born alive per breed and serological status is shown in Table 8.

\section{Discussion}

In this study, we have investigated the association between seropositivity and reproductive performance in sows in the Mekong Delta where JEV is endemic. Of the 315 sows included in the study, $60 \%$ were seropositive, and the risk of being seropositive was increasing with age. A previous study on pigs in the same area showed a similar seroprevalence using HI technique (Thu et al. 2006). The positive correlation between seropositivity and age is in accordance with other studies concerning JEV in humans in endemic areas (Grossman et al. 1973; Bartley et al. 2002) and this report is, to our knowledge, the first time such data for pigs are internationally published.

There was no difference $(p>0.05)$ in the proportion of seropositive sows depending on the month of sampling. This data does not support the notion that season would have a major influence on the risk for a sow to be seropositive. Indeed, a transmission of JEV all year around has been demonstrated in Thailand (Grossman et al. 1974) and since there are favorable conditions for vectors both during the rainy and the dry seasons in the Mekong delta, this is likely to be the case there also, explaining that there were no significant differences between seasons.

The use of an IgG ELISA in this study avoids some of the problems with poor discrimination between different flaviviruses that have been observed with $\mathrm{HI}$ tests (De Madrid and Porterfield 1974). In the Swedish samples, where no flaviviral infection would be expected, it showed a $100 \%$ specificity, although the relative specificity was lower using the data from the manufacturer. To account for the possibility of a titer-dependent association with reproductive performance, the results from the test were used both as a continuous and a categorical variable.

Table 6 Association between serology for Japanese Encephalitis Virus as continuous OD values and the number of piglets born alive in sows $<1.5$ years

\begin{tabular}{lcl}
\hline Effect & $F$ value & $P$ \\
\hline Within breed effect of OD values & 2.72 & 0.0432 \\
Breed & 0.47 & 0.7052 \\
\hline
\end{tabular}


Table 7 Association between serology for Japanese Encephalitis Virus and the number of piglets born alive in sows $<1.5$ years within each breed

\begin{tabular}{lcc}
\hline Breed & Coefficient $^{\mathrm{a}}$ & Standard error \\
\hline Duroc & 10.64 & 12.74 \\
Landrace & -7.53 & 2.48 \\
Yorkshire & -0.44 & 0.71 \\
Hybrids & 1.31 & 1.61 \\
\hline
\end{tabular}

${ }^{a}$ The coefficient shows how a one-unit increase in OD value affects the number of piglets born alive in each breed

In the studies including all sows, the continuous OD values did not show any effect on the number of stillborn piglets and an effect on the number of piglets born alive only when interacting with the effect of the breeds. Seropositivity as such did neither affect the number of piglets born alive nor stillborn. This lack of association could be explained by the high chance for the gilts to achieve a prepubertal protective immunity through natural infection as is the case in infections with Porcine Parvovirus (Mengeling 2006). It has been shown that only the individuals that were immunologically naïve when mated before the epidemics subsequently had more stillborn and mummified fetuses (Morimoto et al. 1972). Maternal protection against JEV has usually disappeared at 46 months of age (Scherer et al. 1959) and a continuous, year-round, transmission of virus from mosquitoes would thus expose the gilts to the risk of infection before pregnancy. Also, a higher infection pressure in the area would lead to a higher proportion of immune gilts. This prepubertal immunity has also been suggested to be the reason why JEV has not been observed to be causing abortions in humans in endemic area (Tsai 2006). Similarly, a correlation between high transmission rate and lower incidence of clinical cases has been observed for Dengue Virus, another mosquito-borne flavivirus (Nagao and Koelle 2008). Indeed, in the analyses of the younger sows, an association between increasing OD values and the number of stillborn piglets could be shown. This suggests

Table 8 Number of piglets born alive in sows $<1.5$ years with different breed and serological status for Japanese Encephalitis Virus

\begin{tabular}{lcc}
\hline & Seronegative & Seropositive \\
\hline Duroc & 9.1 & $\mathrm{NA}^{\mathrm{a}}$ \\
Landrace & 9.3 & 2.8 \\
Yorkshire & 9.0 & 8.7 \\
Hybrid & 10.1 & 10.7 \\
\hline
\end{tabular}

$N A$ nonapplicable

${ }^{\mathrm{a}}$ There were no seropositive duroc in this age category that the highest risk of having negative impacts of JEV on the reproductive performance of the sows is early in life.

Season and age did not influence the reproductive performance by themselves in this study, even though this has been shown in other studies (Dan and Summers 1996; Tantasuparuk et al. 2000).

The breeds Yorkshire and Landrace were negatively associated with increasing OD values in comparison with the Duroc breed and hybrids; however, the standard error was larger than the estimates for Duroc, making these estimates unclear. Breed difference in the impact of JEV on reproduction has, however, been observed before and it has been shown that different strains of mice have very different susceptibility to having the placenta and fetus infected when inoculated with virus during pregnancy (Fujisaki et al. 1976). The results in this study showing the different effects between the breeds may suggest benefits of keeping certain breeds within the tropical areas of the world endemic for JEV but further studies are needed to be able to draw definite conclusions.

In this study, there was an increase of five live piglets born per sow for every one-unit increase in mean OD value for the herd. Considering that only four farms were included in the study, it would be hard to draw definite conclusions from that finding, but a reasonable explanation could be that the immunity of the sows on the farms with a higher mean OD value was better due to a higher infection pressure. The gilts on these farms would thus likely get infected and be immune earlier in life than those on farms with low mean OD value.

\section{Conclusions}

JEV is an emerging disease with severe zoonotic implications and it is considered to be of economic importance for the pig industry (Platt and Joo 2006). The current and potentially enhanced spread of JEV to new areas will render more areas to become endemic in the future. The results in this study are indicative of negative impact of the virus on reproductive performance only in young sows under the endemic conditions common in tropical regions. The results may actually even suggest that a high infection pressure and infection early in life could be beneficiary for the production of piglets in the individual sow. If so, this would imply that reduced reproductive performance would not necessarily be observed in new endemic areas.

Acknowledgements This study was supported by the Swedish International Development Cooperation Agency/Department of Research Cooperation (Sida/SAREC). The authors want to thank Dr. Manh for providing excellent working facilities in Cantho.

Conflicts of interest There were no conflicts of interest during the performance of this study. 
Open Access This article is distributed under the terms of the Creative Commons Attribution Noncommercial License which permits any noncommercial use, distribution, and reproduction in any medium, provided the original author(s) and source are credited.

\section{References}

Bartley, L.M., Carabin, H., Vinh Chau, N., Ho, V., Luxemburger, C., Hien, T.T., Garnett, G.P., Farrar, J., 2002. Assessment of the factors associated with flavivirus seroprevalence in a population in Southern Vietnam, Epidemiology and Infection, 128, 213-220.

Boqvist, S., Thu, H.T.V., Vagsholm, I., Magnusson, U., 2002. The impact of Leptospira seropositivity on reproductive performance in sows in southern Viet Nam, Theriogenology, 58, 1327-1335.

Burns, K., 1950 Congenital Japanese B encephalitis infection of swine, Proceedings of the Society for Experimental Biology and Medicine, 75, 621-625.

Dan, T., Summers, P., 1996. Reproductive performance of sows in the tropics, Tropical Animal Health and Production, 28, 247-256.

De Madrid, A.T., Porterfield, J.S., 1974. The Flaviviruses (Group B Arboviruses): a Cross-neutralization Study, Journal of General Virology, 23, 91-96.

Do, Q.H., Vu, T.Q.H., Huynh, T.K.L., Dinh, Q.T., Deubel, V., 1994. Current situation of Japanese Encephalitis in the south of Vietnam, 1976-1992, Tropical Medicine, 36, 202-214.

Dohoo, I., Martin, W., Stryhm, H., 2009. Veterinary Epidemiologic Research. (VER Inc., Charlottetown, Canada)

Endy, T.P., Nisalak, A., 2002. Japanese encephalitis virus: ecology and epidemiology, Current Topics in Microbiology and Immunology, $11-48$.

Fujisaki, Y., Miura, Y., Sugimori, T., Morimoto, T., Ino, T., 1976. Experimental studies on vertical infection of mice with Japanese Encephalitis-Virus 1. Effect of mouse strain on placental and fetal infection, National Institute of Animal Health Quarterly, 16, 97-106.

Gresham, A., 2003. Infectious reproductive disease in pigs, In Practice, 25, 466-473.

Grossman, R.A., Edelman, R., Willhight, M., Pantuwatana, S., Udomsakdi, S., 1973. Study of Japanese Encephalitis Virus in Chiangmai Valley, Thailand: III. Human seroepidemiology and inapparent infections, American Journal of Epidemiology, 98, 133-149.

Grossman, R.A., Edelman, R., Gould, D.J., 1974. Study of Japanese encephalitis virus in Chiangmia Valley, Thailand. VI. Summary and conclusions, American Journal of Epidemiology, 100, 69-76.

Gubler, D.J., 1996. The global resurgence of arboviral diseases, Transactions of the Royal Society of Tropical Medicine and Hygiene, 90, 449-451.

Hai, L.T., Nguyen, N.H., 1997. Outlines of pig production in Vietnam, Pig News and Information, 18, 91-94.

Hanna, J.N., Ritchie, S.A., Phillips, D.A., Shield, J., Bailey, M.C., Mackenzie, J.S., Poidinger, M., McCall, B.J., Mills, P.J., 1996. An outbreak of Japanese encephalitis in the Torres Strait, Australia, 1995, Medical Journal of Australia, 165, 256-260.

Ho, M.S., Chen, W.J., Chen, H.Y., Lin, S.F., Wang, M.C., Di, J.L., Lu, Y.T., Liu, C.L., Chang, S.C., Chao, C.L., King, C.C., Chiou, J. M., Su, I.J., Yang, J.Y., 2005. Neutralizing antibody response and SARS severity, Emerging Infectious Diseases, 11, 1730-1737.

Hosoya, H., Matumoto, M., Iwasa, S., 1950. Epizootiological studies on stillbirth of swine occurred in Japan during summer months of 1948, Japanese journal of experimental medicine, 20, 587-595.

Hung, I.F.N., To, K.K.W., Lee, C.K., Lin, C.K., Chan, J.F.W., Tse, H., Cheng, V.C.C., Chen, H.L., Ho, P.L., Tse, C.W.S., Ng, T.K., Que,
T.L., Chan, K.H., Yuen, K.Y., 2010. Effect of Clinical and Virological Parameters on the Level of Neutralizing Antibody against Pandemic Influenza A Virus H1N1 2009, Clinical Infectious Diseases, 51, 274-279.

Khasnis, A.A., Nettleman, M.D., 2005. Global warming and infectious disease, Archives of Medical Research, 36, 689-696.

Mengeling, W.L., 2006. In: Straw, B.E., Zimmerman, J.J., D'Allaire, S., Taylor, D.J. (Eds.), Diseases of swine, (Blackwell Publishing Professional, Iowa), 373-387.

Minh, L.Q., Tuong, T.P., van Mensvoort, M.E.F., Bouma, J., 1997. Contamination of surface water as affected by land use in acid sulfate soils in the Mekong River Delta, Vietnam, Agriculture, Ecosystems \& Environment, 61, 19-27.

Morimoto, T., Miura, Y., Sugimori, T., Kurogi, H., Fujisaki, Y., 1972. Isolation of Japanese Encephalitis-Virus and a hemagglutinating DNA virus from brain of stillborn piglets, National Institute of Animal Health Quarterly, 12, 127-136.

Nagao, Y., Koelle, K., 2008. Decreases in dengue transmission may act to increase the incidence of dengue hemorrhagic fever, Proceedings of the National Academy of Sciences, 105, 22382243.

Nguyen, H.T., Nguyen, T.Y., 1995. Japanese encephalitis in Vietnam 1985-1993, Southeast Asian Journal of Tropical Medicine and Public Health, 26, 47-50.

Platt, K.B., Joo, H.S., 2006. Japanese Encephalitis and West Nile Viruses, In: Straw, B.E., Zimmerman, J.J., D'Allaire, S., Taylor, D.J. (Eds.), Diseases of swine (Blackwell Publishing Professional, Iowa), 359-365.

Rosen, L., 1986. The natural history of Japanese Encephalitis Virus, Annual Review of Microbiology, 40, 395-414.

Salmon, H., 1984. Immunity in the fetus and the newborn infant: a swine model, Reproduction, Nutrition, Development, 24, 197206.

Scherer, W.F., Moyer, J.T., Izumi, T., 1959. Immunologic studies of Japanese Encephalitis Virus in Japan: V. Maternal antibodies, antibody responses and viremia following infection of swine, Journal of Immunology, 83, 620-626.

Tantasuparuk, W., Lundeheim, N., Dalin, A.M., Kunavongkrit, A., Einarsson, S., 2000. Reproductive performance of purebred Landrace and Yorkshire sows in Thailand with special reference to seasonal influence and parity number, Theriogenology, 54, 481-496.

Tardei, G., Ruta, S., Chitu, V., Rossi, C., Tsai, T.F., Cernescu, C., 2000. Evaluation of Immunoglobulin M (IgM) and IgG Enzyme Immunoassays in Serologic Diagnosis of West Nile Virus Infection, Journal of Clinical Microbiology, 38, 2232-2239.

Thu, H.T.V., Thu, L.T., Phuc, N.D., Tu, T.D., Trang, H.N., 2006. Prevalence of Japanese encephalitis virus (JEV) in breeding pig herds in some provinces of Mekong Delta, Veterinary sciences and technique (in Vietnamese), XIII, 73-77.

Tsai, T.F., 2006. Congenital Arboviral Infections: Something New, Something Old, Pediatrics, 117, 936-939.

Vo-Tong, X., Le Than, H., Chau, B.L., 1995. Research priorities for improving animal production by agro-ecological zone in Vietnam, In: Devendra, C., Gardiner, P. (Eds.), Global Agenda for Livestock Research Proceedings of the Consultation for the South-East Asia Region, International Livestock Research Institute, 10-13 May 1995 (IRRI, Los Banos, The Philippines)

Xinglin, J., Huanchun, C., Xiang, W., Changming, Q., 2005. Quantitative and Qualitative Study of Enzyme-linked Immunosorbent Assay to Detect IgG against Japanese Encephalitis Virus in Swine Sera, Veterinary Research Communications 29, 159-169. 